\title{
A Quadratura do Retângulo no Códice Atlântico DE LEONARDO DA VINCI
}

\author{
Jeová Pereira Martins \\ Secretaria de Estado de Educação do Pará - SEDUC-PA. Brasil \\ Iran Abreu Mendes \\ Universidade Federal do Pará - UFPA - Brasil
}

(aceito para publicação em outubro de 2021)

\begin{abstract}
Resumo
O presente artigo relata parte dos resultados de uma pesquisa mais ampla e tem como objetivo discutir o método de Leonardo da Vinci para a quadratura do retângulo no Códice Atlântico. Trata-se de um manuscrito que contém 1.119 folhas de desenhos e anotações sobre temas abordados por Da Vinci. Neste artigo, analisamos a folha 544r na qual Da Vinci demonstra, por meio de desenhos e anotações, como transformar um retângulo em um quadrado de área equivalente. O texto resulta de uma pesquisa de cunho qualitativo que tomou o manuscrito como fonte histórica de informações na perspectiva de Barros (2004). Os resultados comprovam que Da Vinci demonstrou a quadratura do retângulo e mobilizou, para isso, a geometria de Os Elementos de Euclides, relacionada às suas técnicas de desenho, o que culminou em uma geometria prática por ele desenvolvida ao longo de sua vida como artista e cientista.
\end{abstract}

Palavras-chave: Da Vinci, Códice Atlântico, Quadratura do Retângulo, História da Matemática.

\section{[THE SQUARE OF THE RECTANGLE IN LEONARdo da Vinci's CODEX ATLANTIC]}

\begin{abstract}
This article reports part of the results of a broader research and aims to discuss Leonardo da Vinci's method for squaring the rectangle in the Atlantic Codex. It is a manuscript containing 1.119 sheets of drawings and notes on topics covered by Da Vinci. In this article we look at sheet 544r on which Da Vinci demonstrates, through drawings and annotations,
\end{abstract}

RBHM, Vol. 21, n 41, pp. 73-88, 2021 
how to transform a rectangle into a square of equivalent area. The text is the result of a qualitative research that took the manuscript as a historical source of information from the perspective of Barros (2004). The results prove that Da Vinci demonstrated the squareness of the rectangle and mobilized the geometry of The Elements of Euclid for this purpose, related to his drawing techniques, which culminated in a practical geometry developed by him throughout his life as an artist and scientist.

Keywords: Da Vinci. Atlantic Codex. Square of the Rectangle. History of Mathematics.

\section{Introdução}

O presente artigo apresenta uma parte dos resultados de uma pesquisa mais ampla que estudou o Códice Atlântico, que é um manuscrito de Leonardo da Vinci que reúne desenhos e anotações que versam sobre temas como anatomia, óptica, ciência dos pesos, balística, arquitetura e geometria. $\mathrm{O}$ recorte da pesquisa realizada centrou-se nas relações dos desenhos do Códice Atlântico com a geometria da Educação Básica e identificou desenhos que remetem a temas como Teorema de Pitágoras, formas geométricas planas (triângulo, retângulo, pentágono, hexágono, círculo...), formas geométricas sólidas, medição de áreas etc. Com esse enfoque, o manuscrito foi tomado como uma fonte textual histórica a ser investigada segundo a perspectiva da fonte como discurso, conforme propõe Barros (2004), ou seja, a fonte como portadora de informações em si, cujo texto (no sentido ampliado) é abordado qualitativamente na busca por informações não apenas na superfície, mas que estão submersas em suas camadas complexas, como as que se referem aos contextos histórico e sociocultural, nos quais as informações contidas na fonte foram produzidas.

É, portanto, com esse pensamento que neste artigo abordamos um estudo interpretativo sobre uma das folhas do referido Códice na qual Da Vinci transforma um retângulo em um quadrado de área equivalente, procedimento que já se realizava na Grécia Antiga e era conhecido como quadratura do retângulo (KLINE, 1992; KATZ, 2010). Assim discutiremos o método de Da Vinci para a quadratura do retângulo no Códice Atlântico.

O Códice Atlântico é composto por 1119 folhas e a edição que pesquisamos, publicada no Brasil, é constituída por 10 volumes que contemplam 602 folhas as quais estudamos na pesquisa mais ampla. A folha em estudo neste texto, 544r, tem como foco a conservação da área por meio da transformação de uma figura plana em outra de área equivalente, tema recorrente no Códice Atlântico. Essas transformações interessavam a Da Vinci por fornecerem analogias para sua Arte, principalmente a pintura, área na qual se destacou, pois tem sob sua autoria obras como A última ceia, Batismo de cristo e Mona lisa. Cada volume da edição do Códice estudada contém um bloco de folhas com desenhos e anotações de Da Vinci contendo reto e verso (rv) e, ao final do bloco, páginas com um aparato crítico que contém a descrição de cada folha, em português, a transcrição das anotações de Da Vinci feita por Algusto Marinoni em italiano arcaico e algumas notas explicativas sobre os desenhos e os temas abordados. $\mathrm{O}$ aparato crítico se refere a cada uma 
das folhas do bloco e foi fundamental para a leitura em compreensão dos desenhos e anotações de Da Vinci.

Assim, no estudo da folha 544r, consideramos os desenhos do reto e do verso, fizemos a leitura da descrição e fizemos uma tradução livre dos textos de Da Vinci transcritos por Marinoni e estudamos Os Elementos de Euclides, tendo em vista que Da Vinci utilizou duas proposições do livro I e uma definição do livro II de Os Elementos na demonstração da quadratura do retângulo. Esse fato evidencia que os estudos de Da Vinci mobilizavam a geometria Euclidiana em uma relação entre essa geometria e a Arte o que tangencia tanto a história da matemática como a história da Arte, principalmente a do Renascimento, época fértil e revolucionária em que Da Vinci viveu.

\section{Leonardo da Vinci e o Códice Atlântico}

Da Vinci nasceu na cidade de Vinci, na região da toscana, na Itália do Renascimento. Segundo registro de seu avô, Antônio, o nascimento se deu em um sábado, 15 de abril de 1452 às 10:30 da noite, registro esse perdido em um caderno de notas após a morte de Da Vinci e encontrado em 1939. Filho ilegítimo de Ser Piero da Vinci (tabelião em Florença) com uma camponesa de Vinci chamada Caterina, foi criado pelos avós no lugar onde nasceu. Apesar de a mãe residir na mesma cidade, há poucos indícios de que ele tenha convivido com ela (WHITE, 2002).

Na década de 1460, com o falecimento dos avós, Da Vinci foi morar com o Pai em Florença e iniciou seus estudos na oficina de Andrea del Verrocchio (1435-1488), um mestre-artesão da cidade. Da Vinci permaneceu nessa oficina (bottega) por mais ou menos 6 anos obtendo o registro de mestre-artesão por volta de 1472. A partir daí iniciou profissionalmente seus trabalhos como artista, arquiteto e engenheiro, trabalhos geralmente encomendados por governantes e líderes religiosos da época (WHITE, 2002).

A obra de Da Vinci é volumosa. Após sua morte, em 23 de abril de 1519, em Amboise, na França (no solar Cloux), onde ele exerceu as funções de Pintor, Arquiteto, Engenheiro e Mecânico da corte do Rei Francisco I, seus estudos e anotações foram herdados por seu amigo Francesco Melzi. Tais anotações constituíam uma coleção com cerca de 13 mil páginas, das quais, atualmente, conhece-se o paradeiro de mais ou menos 7 mil, boa parte delas reunidas em Códices em bibliotecas da Itália e de outros Países. Dentre essas coleções, está o Códice Atlântico ${ }^{1}$, que reúne estudos e desenhos de diferentes áreas de conhecimento pelas quais Da Vinci transitava com excelência (ISAACSON, 2017; WHITE, 2002).

O referido Códice é formado por 1119 folhas (em torno de 1750 fragmentos, pois há folhas que possuem mais de um fragmento como a 56, que se subdivide em $56 \mathrm{a}, 56 \mathrm{~b}$, 56c, 56d e 56e) que tratam de anotações de Da Vinci reunidas em um único volume por Pompeo Leoni no final do século XVI, em Milão, e que passou por processos de restauração e de reorganização até chegar ao formato atual que se encontra na Biblioteca Ambrosiana de Milão. Recebe o nome de Códice Atlântico por se tratar de um manuscrito (códex) em folhas no formato atlântico, ou seja, de dimensões $65 \mathrm{~cm} \mathrm{x} 44 \mathrm{~cm}$ e pela

1 o Códice Atlântico é a maior coleção de desenhos e estudos de Leonardo da Vinci.

RBHM, Vol. 21, n 41, pp. 73-88, 2021 
inscrição que aparece na capa da encadernação original do século XVI (SÁNCHEZ; ALMARZA, 2008).

“DISEGNI DI MACHINE ET / DELLE ARTI SECRETI . ET ALTRE COSE / DI LEONARDO DA VINCI / RACOLTI DA/ POMPEO LOE / NI' (Desenhos de máquinas e de artes secretas e outras coisas de Leonardo da Vinci recompilados por Pompeo Leoni) (SÁNCHEZ; ALMARZA, 2008, p. 6-7).

O velho Códice passou por alguns percalços até a atualidade. Após a morte de Pompeo Leoni, um de seus herdeiros vendeu o códice para o marques Galeazzo Arconati, pertencente à aristocracia milanesa, que o doou para a Bibliteca Ambrosiana em 1637. Porém, em 1796, o Rei Napoleão da França conquistou a região da Lombardia (consequentemente Milão) e ordenou que obras da Arte e da Ciência fossem levadas para a França, dentre elas o Códice Atlântico, que ficou na Biblioteca Nacional de Paris. No entanto, com a queda de Napoleão, decidiu-se, no congresso de Viena em 1815, que as obras artísticas deveriam ser devolvidas aos países conquistados. Assim, o códice retornou para a Biblioteca Ambrosiana, onde permaneceu até a década de 1960 quando precisou ser transportado para Roma, a fim de ser restaurado.

O processo restauro que se deu nas décadas de 1960 e 1970 implicou na desencadernação do velho Códice e reencadernação em 12 volumes com os desenhos, aos quais se acrescentaram outros 12 volumes de transcrições e aparatos críticos de Algusto Marinoni. Essa edição foi reproduzida (em Fac-símile) em 998 exemplares (destinados e adquiridos por Bibliotecas e pesquisadores) que, por sua vez, foram editados por todo o mundo em diferentes formatos, alguns com a totalidade das folhas e outros com parte, apenas (NAVONI, 2012).

Foram manifestadas críticas da comunidade científica pelo fato de que, quando exposto ao público, só se tinha acesso a 12 desenhos, uma vez que cada um dos 12 volumes era aberto em uma determinada página e colocado em local que só permitia a visualização, não sendo possível a manipulação, fato que restringiu o acesso ao conteúdo do Códice. Por esse motivo, em 2009, o Códice foi desencadernado e as mais de 1000 folhas foram colocadas separadamente em quadros que as protegem, mas permitem a consulta (NAVONI, 2012).

Dentre as tantas edições do Códice Atlântico produzidas pelo mundo, a da Editora Fólio de Barcelona (em fac-símile) contém todas as 1119 folhas em 20 volumes, com descrição, transcriçõos e os aparatos críticos de Algusto Marinoni. Desta edição, foi organizada uma edição brasileira, sob direção de José Luis Sànchez e Meritxell Almarza e tradução das descrições de cada folha para o português de Carlos Nougué, que conta com 10 volumes que contemplam as 602 primeiras folhas do Códice Atlântico. Esta última edição foi a que estudamos na pesquisa ampliada, da qual fizemos o recorte para este texto.

O Códice Atlântico reúne uma coleção de documentos que abarcam praticamente toda a vida artística e científica de Da Vinci, produzidos por ele no período de 1478 a 1519 (41 anos, aproximadamente). Contém estudos e apontamentos práticos e teóricos sobre temas como: arquitetura, arte da guerra, mecânica, astronomia, hidráulica, óptica, anatomia, 
botânica, zoologia, estudos sobre o voo, estudos sobre a água, textos literários, anotações autobiográfica, perspectiva, aritmética e geometria, este último foco de nossa pesquisa (ISAACSON, 2017; NAVONI, 2012).

\section{A quadratura do retângulo por Leonardo da Vinci}

Dentre os estudos de geometria feitos por Da Vinci, destacamos as transformações de um sólido geométrico em outro de mesmo volume ou de uma figura plana em outra de mesma área. Os estudos sobre essas transformações remontam a Antiguidade Grega e se relacionam com os problemas da quadratura do círculo e da duplicação do cubo. Hipócrates de Chios (460 a.C.-370 a.C.) foi um dos geômetras gregos que se dedicou a quadrar o círculo e desenvolveu o primeiro método para se calcular a área exata de uma figura curva, pois, segundo sua lei, "quando se cria uma lúnula ao sobrepor um grande semicírculo sobre outro menor, pode-se desenhar um triangulo reto dentro do maior que terá mesma área da lúnula" (ISAACSON, 2017, p. 233; OZANAM, 1814).

Da Vinci se interessava pela geometria das transformações por ela fornecer analogias para sua arte, principalmente suas pinturas, nas quais buscava retratar corpos em movimento. Tais pinturas eram verdadeiras narrativas de cenas e acontecimentos e por isso foram classificadas como pinturas narrativas ou pinturas psicológicas, pois objetivavam transmitir as emoções dos personagens retratados (angústia, felicidade, surpresa, indignação, súplica, temor, paixão etc.). Para tanto, Da Vinci detalhava ao extremo os corpos em movimento: braços contorcidos, olhares e sorrisos misteriosos, rostos virados, pessoas ajoelhadas, montadas a cavalo e em combate, além de retratar a paisagem geográfica e climática (névoa, rochedos, flores e plantas) e a água em movimento, por exemplo. Esse detalhamento, por vezes obsessivo, pode ser observado em obras como a Última Ceia, Dama com arminho, Virgem dos rochedos, Anunciação, O batismo de cristo e o São Jerônimo no deserto, retratado na figura 1 (ISAACSON, 2017).

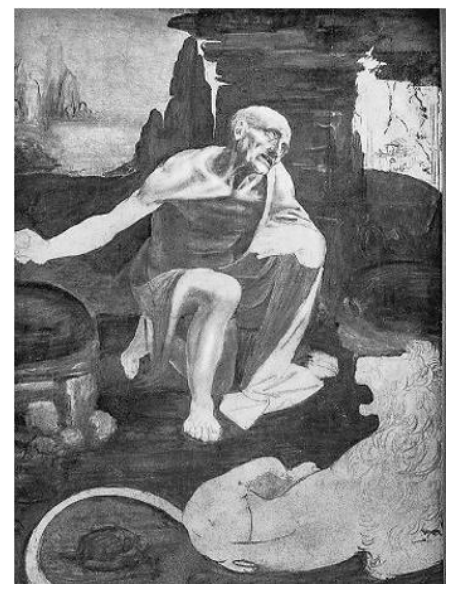

Figura 1: São Jerônimo no deserto Fonte: Isaacson, 2017, p. 105. 
A analogia entre a geometria das transformações e a pintura de Da Vinci se estabeleceu quando ele percebeu que um corpo que se movimenta, se desloca, mantém seu volume inalterado, ou seja, muda somente a forma, o que ocorre analogamente quando se transforma uma figura geométrica plana em outra de forma diferente, mas com a mesma área. Como seu foco era a conservação do volume dos corpos, estendeu seus estudos para as transformações volumétricas de sólidos geométricos como pirâmides, cilindros, cubo e esfera. Por outro lado, Da Vinci buscava fundamentar sua arte cientificamente e o fazia por meio da geometria pois tinha a matemática como aquela que conferia o status de ciência verdadeira a todo estudo realizado (A VIDA, 1971; ISAACSON, 2017).

No Códice Atlântico, boa parte dos desenhos de Da Vinci são oriundos de seus estudos sobre a geometria das transformações. Nos 10 volumes que estudamos, identificamos em torno de 180 folhas contendo desenhos sobre o tema. São desenhos sobre quadratura do círculo, duplicação do cubo, lúnulas, transformação de um sólido geométrico em outro e transformações entre áreas. Neste último, nosso foco neste artigo, analisamos a folha 544rv (figura 2), que trata da quadratura do retângulo.

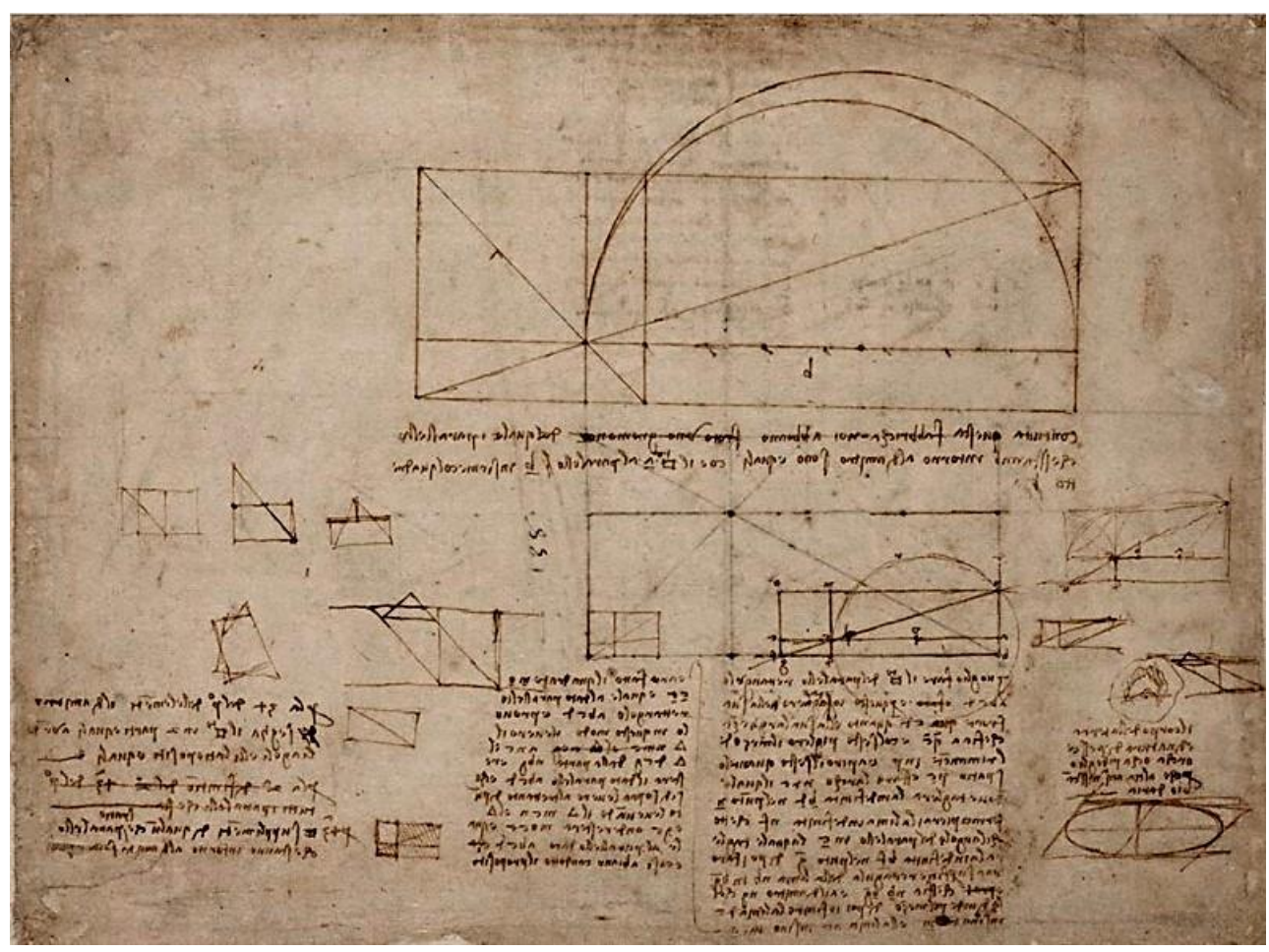

Figura 2: folha $544 \mathrm{r}$

Fonte: Sánchez e Almarza (2008). 
Em nosso estudo, apresentamos, inicialmente, a folha completa com uma descrição suscinta; destacamos partes da folha que jugamos importantes para a compreensão dos desenhos e utilizamos a tradução dos textos de Da Vinci transcritos, com vistas a compreender e também explicar aos leitores o método utilizado por Da Vinci no seu processo de quadratura do retângulo. Na folha 544r, Da Vinci desenvolve a quadratura do retângulo a partir das Proposições 34 e 43 do Livro I e da Definição 2 do Livro II de Os Elementos.

A folha apresenta um grupo de desenhos geométricos (em torno de 14) e a maioria trata da quadratura do retângulo. Na parte superior central da folha, o desenho maior é um retângulo, sobre o qual são traçados segmentos de retas horizontais, verticais e diagonais que o subdividem em retângulos e triângulos. Há, ainda, dois arcos de circunferência na parte superior. Um grupo de desenhos menores abaixo parecem reproduzir o desenho maior e outros parecem ser partes originadas pela sua divisão. No canto inferior direito da folha, há um desenho oval que parece não ter relação com os outros desenhos da folha.

Pode-se identificar, ainda, a presença de escritos que se dividem em cinco grupos: um abaixo do desenho maior, na metade superior da folha, e outros quatro em colunas verticais na metade inferior da folha. $O$ texto de cada grupo parece ter relação com os desenhos que ficam próximos a ele, o que nos levou a inferir conclusões após o estudo das transcrições e a tradução (livre), que nos ajudaram a compreender como Da Vinci procedeu para fazer a quadratura do retângulo. Além disso, os desenhos e os escritos de Da Vinci nessa folha mostram como ele associava a demonstração escrita à geométrica, uma vez que a ferramenta que ele dominava era o desenho. Dos cinco grupos de textos, destacamos, inicialmente, o que está na terceira coluna da esquerda para a direita, juntamente com o desenho a que se refere (figura 3).

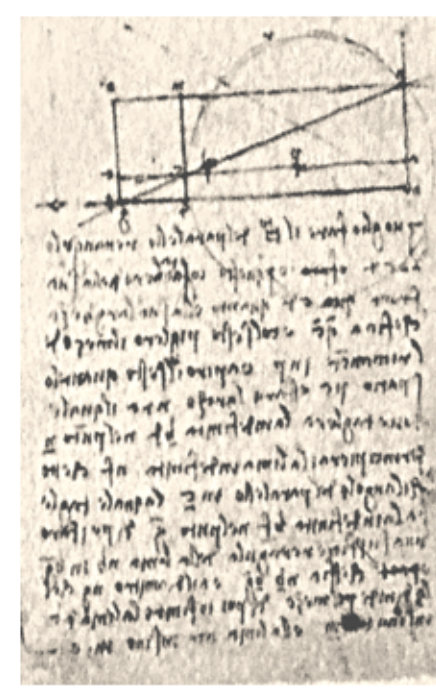

Quero traçar um quadrado a partir do retângulo a $b$ c $d$ $e$, para isso, cortarei (na frente $c \boldsymbol{d}$, a qual é a largura) que é para a q c; e com o compasso, tomarei a metade do segmento restante em $p$, e abrirei o compasso com medida pc, e fareio arco ncr, que cortaráo bd indefinido no ponto $n$, você definirá a linha indefinida nf, que toca o ângulo do paralelo eme, que cortaráo bf indefinido no ponto $g$. Então farei um retângulo na superfície da linha $n \boldsymbol{b}$ em $\boldsymbol{b} g$, que é $\boldsymbol{n} \boldsymbol{b}$ o $g$, e tenho o diâmetro $n g$ que o divide pela metade. Então traçarei a linha $\mathbf{a}$ c até $\boldsymbol{m}$, e a linha a c até e, e o quadrado mo ce, é igual ao retângulo

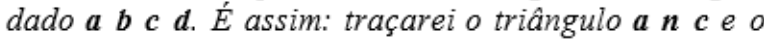
triangulo $\boldsymbol{d} \boldsymbol{c} \boldsymbol{g}$ da parte $\boldsymbol{n} \boldsymbol{b} \boldsymbol{g}$, e o paralelo dado permanecerá $\boldsymbol{a} \quad \boldsymbol{b} \quad \boldsymbol{c} \quad \boldsymbol{d}$. E assim, acima, levantarei $o$ máximo de pontos, elevando o triângulo $m \boldsymbol{c} n$ e o triângulo e $g \mathrm{c}$, de modo que restará $m$ o $c$ e igual ao paralelo dado $\boldsymbol{a}$ b $c$ c $d ;$; $e$ assim eles conduzirão $o$ propósito.

Figura 3: procedimento de Da Vinci para quadrar o retângulo Fonte: Sánchez e Almarza (2008, p. 153-154).

RBHM, Vol. 21, n 41, pp. 73-88, 2021 
O destaque se deu por ser esse texto aquele em que Da Vinci descreve com mais completude a demonstração que fez, ao passo que nos demais ele escreve apenas fragmentos às vezes incompletos, embora estes, também, sirvam para compor a ideia central. O desenho, por mais que seja menor que aquele colocado no centro da folha, é o que apresenta os vértices e outros pontos nomeados, como os pontos $\mathbf{n}, \mathbf{m}, \mathbf{o}$, no lado horizontal superior, e os pontos b, d, g, no lado horizontal inferior do retângulo maior. A Figura 3 apresenta: o desenho; os escritos de Da Vinci, logo abaixo; e a tradução livre que fizemos a partir da transcrição de Algusto Marinoni.

Após o trabalho de observação e estudo do texto transcrito, da tradução que fizemos, da sua correlação com os desenhos e com o método adotado por Da Vinci para quadrar o retângulo e do estudo de Os elementos, fundamento adotado por ele na demonstração, identificamos no texto duas incoerências do ponto de vista matemático, quando posto em correspondência com o desenho, pois dois pontos da figura, que foram identificados por letras minúsculas (pontos e e n), não estão localizados nela como o texto informa. A partir de nossa interpretação do desenho e da demonstração de Da Vinci no texto escrito, chegamos à conclusão de que o retângulo que se quer quadrar é o retângulo abcd (figura 4), que será usado para que se esclareçam as incoerências apontadas. O ponto q é a medida da altura do retângulo que foi subtraída por Da Vinci do segmento ac para que fosso obtido aq e, posteriormente o seu ponto médio p. O segmento pc, por sua vez, será o raio do arco que irá determinar a medida do lado do quadrado que se deseja encontrar.

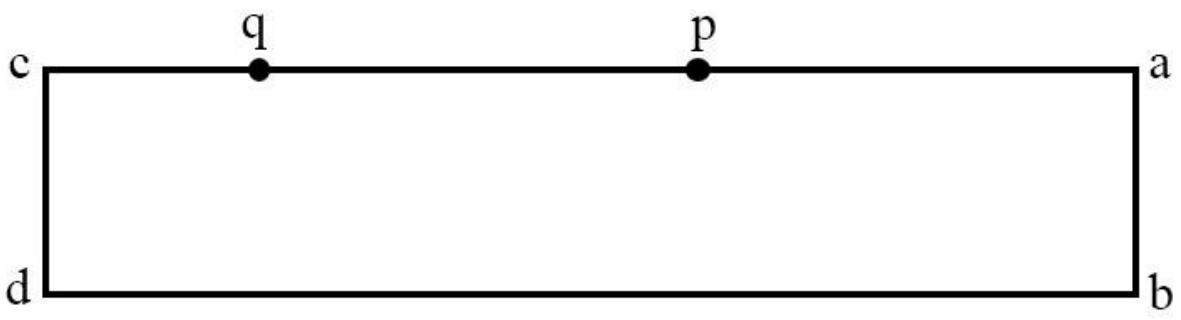

Figura 4: retângulo abcd

Fonte: Elaborado a partir de Sánchez e Almarza (2008).

A prmeira incoerênica que encontramos está na frase farei o arco ncr, que cortará $o$ bd indefinido no ponto $n$. Nela, Da Vinci descreve o procedimento que tomou para traçar um arco que irá contar o prolongamento do lado bd, do retângulo abcd, no ponto n. Tal arco tem raio pc (figura 4) e é traçado a partir do ponto c no sentido anti-horário (figura 5) em direção ao prolongamento do lado esquerdo vertical ba, do retângulo abcd, intersectando-o no ponto n. Assim, o ponto n é o ponto de intersecção do arco com o prolongamento do lado ba e não deste com bd, como está escrito na transcrição do texto.

A seguir, no texto menciona-se a frase: você definirá a linha indefinida $\boldsymbol{n f}$, que toca o ângulo do paralelo em e, e cortará o bf indefinido no ponto $\boldsymbol{g}$ ”. Novamente, encontramos uma incoerência, pois a linha nf é a diagonal (figura 5) que parte de $\mathbf{n}$ e toca o vértice c do retângulo abcd. Essa diagonal não passa pelo ponto e, pois mais adiante Da 
Vinci se refere ao ponto e como a intersecção do prolongamento do lado ac com o lado fo do retângulo maior, o que o localiza em outro lugar do desenho, qual seja o vértice do segmento ce, que é lado horizontal inferior do quadrado moec. Informamos que os pontos $\mathbf{a}, \mathbf{b}, \mathbf{c}, \mathbf{e}, \mathbf{d}$ etc. que constam em nossa tradução são os mesmos que estão na transcrição do texto de Da Vinci, e que os colocamos em minúsculas por estarem assim no referido texto.

Tais incoerências podem ter ocorrido por fatores como: problemas com a tradução que fizemos; equívocos cometidos na transcrição dos manuscritos de Da Vinci, o que é compreensível por se tratar de uma escrita do Século XV e feita da direita para a esquerda, como já se sabe da escrita de Da Vinci; ou por ele ter trocado, intencionalmente, algumas letras que se referem a pontos do desenho para manter seu método em sigilo, o que para Isaacson (2017) era sua prática para que niguém copiasse suas descobertas e inventos.

Para que o procedimento de Da Vinci, na quadratura do retangulo, ficasse mais compreensível, utilizamos a figura maior da folha em estudo, marcamos nela os pontos (agora com letras maiúsculas) de forma que houvesse uma coerência entre o desenho, o texto transcrito e o método usado na demostração e escrevemos um texto explicativo do procedimento. Reinteramos que as alterações que fizemos ocorreram com base na transcrição do texto do próprio Da Vinci e de nossa compreensão dos procedimentos tomados por ele na demonstração. A figura 5 destaca o desenho que melhor representa a quadratura do retângulo feita por ele.

A partir do desenho, é possivel identificar o retângulo que se quer quadrar $\mathrm{ABCD}$ e o quadrado MOEC de mesma área obtido no procedimento, ambos internos ao retângulo maior BNOG. Destacamos, ainda, os pontos $\mathrm{Q}(\mathrm{CQ}=\mathrm{CD})$, o ponto $\mathrm{P}$ e o ponto $\mathrm{N}$, importantes para que se compreenda a demostração de Da Vinci que inicialmente traçou um semicírculo AC que não serviu para a demostração.

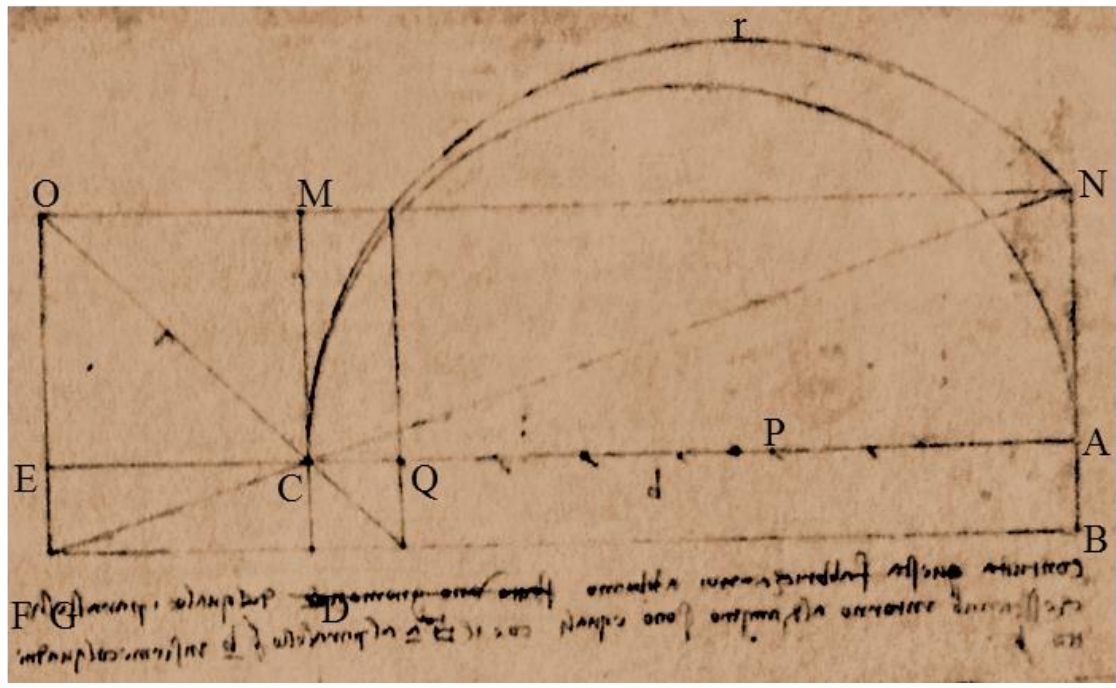

Figura 5: Desenho de Da Vinci para a quadratura do retângulo Fonte: Elaborado a partir de Sánchez e Almarza (2008).

RBHM, Vol. 21, n 41, pp. 73-88, 2021 
Da Vinci deixa clara sua intenção de fazer um quadrado do retângulo ABCD e inicia os procedimentos. Ele mede a altura DC do retângulo e subtrai essa medida do lado $\mathrm{AC}$, a partir de $\mathrm{C}$, marcando o ponto Q. Assim, obtém o segmento CQ que tem a mesma medida da altura DC. Observe o segmento AC na figura 3. Ao subtrair o segmento CQ de $\mathrm{AC}$, resta o segmento AQ. Da Vinci encontra, então, o ponto médio $\mathrm{P}$ do segmento restante AQ. Centra o compasso em P, com abertura até C. Com essa abertura, traça o arco de circunferência $\mathrm{NCr}$, que vai de $\mathrm{C}$, no sentido anti-horário, até o ponto $\mathrm{N}$, que é intersecção do referido arco com o prolongamento do lado vertical direito do retângulo ABCD, ou seja, o lado BA. O segmento AN encontrado corresponde ao lado do quadrado que se quer encontrar. Da Vinci traça a linha NF, que passa por C, e a prolonga até que ela intersecte o prolongamento do lado BD no ponto G. Em seguida, ele constrói o retângulo que tem como lados os segmentos NB e BG, que chama de NBOG de diâmetro NG.

Até aqui, ainda não ficou claro o suficiente o método de Da Vinci para quadrar o retangulo $\mathrm{ABCD}$. Com vistas a compreender e explicar da melhor forma possível tal método e seus procedimentos, recriamos o desenho feito por ele (figura 6). Tentamos seguir os procediemtos de Da Vinci utilizando, porém, a linguagem matemática atual.

Os procedimentos que adotamos resultaram na figura 6 e podem ser seguidos por quem decidir refazer os passos de Da Vinci na quadratura do retângulo.

1. Construa um retângulo $\mathrm{ABCD}$ de lados quaiquer;

2. Meça com o compasso a altura $(\mathrm{CD})$ do retângulo;

3. Mantendo-se a abertura CD, fixe o compasso em $\mathrm{C}$ e "corte" o lado AC no ponto Q. Assim, CQ = CD;

4. Considere, agora o segmento restante AQ e obtenha o seu ponto médio P.

5. Fixe o compasso no ponto $\mathrm{P}$ com abertura até o ponto $\mathrm{C}$;

6. Mantenha a abertura e trace um semicírculo com centro em P, no sentido antihorário, a partir de C;

7. Prolongue o lado vertical direito (BA) do retângulo $\mathrm{ABCD}$ até intersectar o semicírculo e denomine este ponto de intersecção de $\mathrm{N}$. O segmento AN corresponde à medida do lado do quadrado que se pretende construir. Com essa medida, já é possivel construir um quadrado, com régua e compasso, com área equivalente à área do retângulo ABCD. No entanto, Da Vinci segue a demostração e falaremos sobre isso mais adiante. Assim, seguimos com ele;

8. Trace uma semirreta NC, a partir de $\mathrm{N}$;

9. Prolongue o lado horizontal (BD) do retângulo ABCD até intersectar a semirreta NC. Chame esse ponto de intersecçãp de G;

10. Trace uma perpendicular passando por $\mathrm{G}$; 
11. Meça com o compasso o lado $\mathrm{BN}$ e com essa medida, na perpendicular, obtenha o segmento GO, paralelo a $\mathrm{BN}$;

12. Traçe o segmento NO. Você obteve o retângulo NBOG;

13. Prolongue o segmento DC até intersectar NO e marque o ponto M de intersecção;

14. Prolongue $\mathrm{AC}$ até intersectar $\mathrm{OG}$ e marque o ponto de intersecção $\mathrm{E}$.

Com esse passo a passo e com a construção da figura 6 , julgamos se tornar mais compreensível o procedimento de Da Vinci para quadrar o retângulo. Resta, porém, fazermos a correspondência entre a demostração de Da Vinci, a figura que construímos a partir dela e os fundamentos de Euclides, que Da Vinci utilizou para dar sustentação teórica à sua demostração. Assim, retomaremos a demonstração para explicitarmos a prova que Da Vinci obteve, de que o quadrado contruído tem área equivalente ao retângulo inicial ABCD.

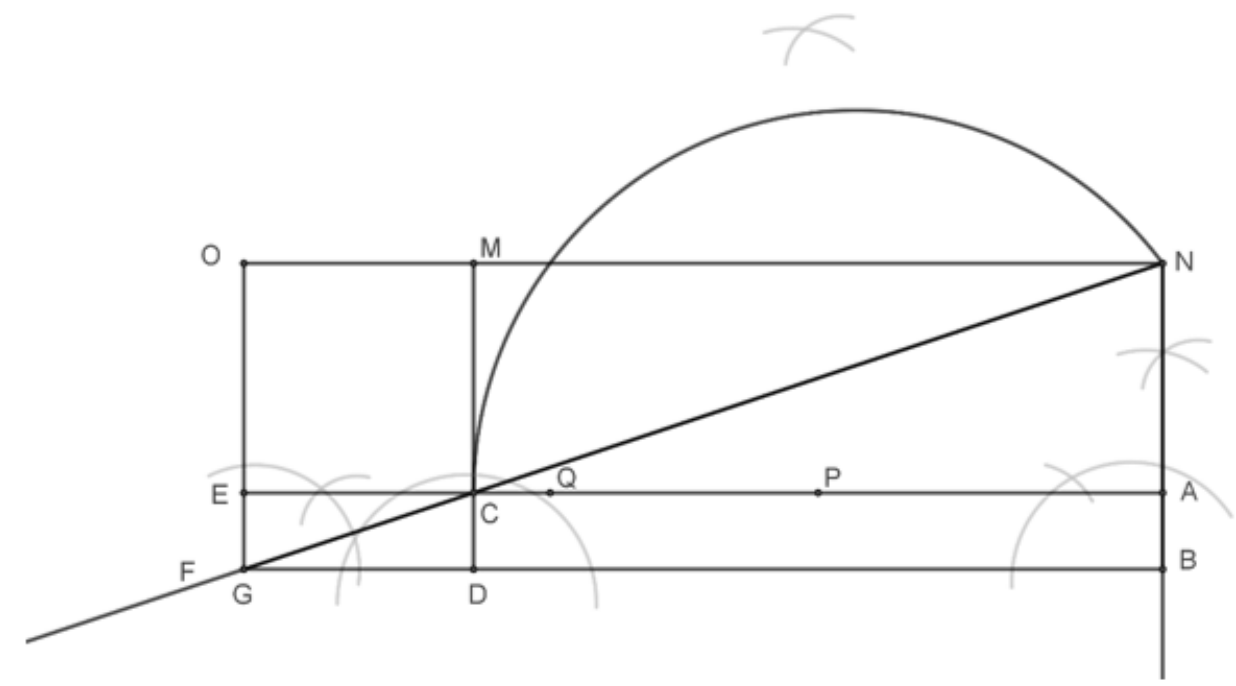

Figura 6: Desenho com régua e compasso

Fonte: Elaborado a partir de Sánchez e Almarza (2008, p. 153-154).

Quando traça o segmento ng, Da Vinci diz: tenho o diâmetro ng que o divide pela metade. Ou seja, ele afirma que o diâmetro NG divide o retângulo maior NBOG em duas partes de mesma área. Ele faz essa afirmação embasado na Proposição 34 do Livro I de $O s$ Elementos, pois em outro fragmento de texto da folha em estudo ele diz: Pela trigésima quarta do primeiro livro dos Elementos, o diâmetro divide o quadrado em duas partes iguais, tendo os ângulos e lados opostos iguais, o que está de acordo com o texto do livro de Euclides referente a tal proposição: duas áreas paralelogrâmicas, tanto os lados quanto RBHM, Vol. 21, n 41 , pp. 73-88, 2021 
os ângulos opostos são iguais entre si, e a diagonal corta-as em duas (EUCLIDES, 2009, p. 124).

A partir dessa proposição, pode ser comprovada a validade da demonstração de Da Vinci pois, observando a figura 6, pode-se verificar que o retângulo NBOG é dividido em dois triângulos maiores NBG e NOG que, por Euclides I 34, têm mesma área. Observe que o triângulo NBG se subdivide em três porções: o triângulo NAC, o triângulo CDG e o quadrilátero $\mathrm{ABCD}$. $\mathrm{O}$ triângulo NOG também se subdivide em três porções: o triângulo NMC, o triângulo CEG e o quadrilátero MOEC.

Ocorre que os triângulos NAC e NMC têm mesma área por serem obtidos pela diagonal NC do quadrilátero ANMC. Como os triângulos CEG e CDG são obtidos pela diagonal do quadrilátero DCEG, também possuem mesma área. Assim, como já se sabe, a partir de Euclides I 34, que os triângulos NBG e NOG têm mesma área, conclui-se que os quadriláteros $\mathrm{ABCD}$ e MOEC também têm mesma área. Portanto, o procedimento de Da Vinci resultou na construção de um quadrado (MOEC) de área equivalente à área de um retângulo $(\mathrm{ABCD})$.

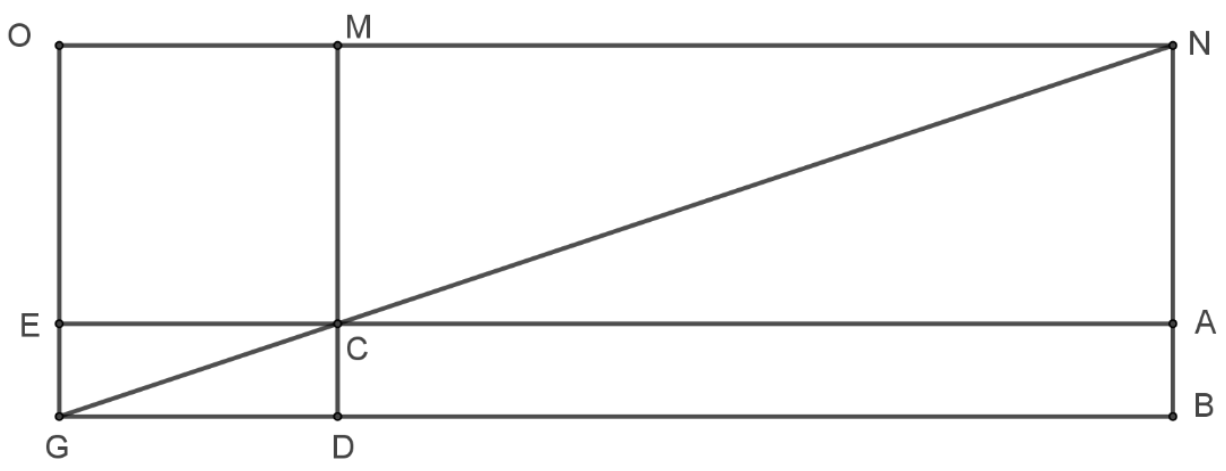

Figura 7: retângulo NBOG

Fonte: Elaborado a partir de Sánchez e Almarza (2008, p. 153-154).

Da Vinci foi um estudioso dedicado que não se contentou com a superficialidade dos temas que estudou. Ele se aprofundava tanto nos estudos que, por vezes, era traído por sua mente curiosa e perdia o foco inicial. Porém, não se sabe ao certo até que ponto Da Vinci estava interessado em buscar analogias na geometria para sua arte ou se interessava em aprender e praticar a geometria na busca por conhecê-la melhor. Após estudar parte de seus desenhos no Códice Atlântico e algumas biografias a seu respeito, podemos julgar que ele buscava o conhecimento para sua arte, mas que ia além e se dedicava, também, à geometria por prazer, curiosidade e vontade de conhecer.

Um exemplo de seu aprofundamento é o fato de que, na demonstração que ora estudamos, ele poderia tê-la interrompido quando traçou o arco $\mathrm{NCr}$ e encontrou o ponto $\mathrm{N}$, uma vez que o segmento AN corresponde ao lado do quadrado que queria construir. Da Vinci segue a construção, pois quer, ainda, verificar a utilização das proposições de 
Euclides em sua construção. Como já comprovamos, a proposição 34 foi suficiente para provar a equivalência entre as áreas do retângulo e do quadrado. Não contente, Da Vinci evoca, também, a proposição 43 do livro I de Os elementos, segundo a qual: Os complementos dos paralelogramos, à volta da diagonal de todo paralelogramo, são iguais entre si. No exemplar que adquirimos de Os elementos, a proposição 43 está acompanhada do desenho retratado na figura 8 .

Considerando a figura 8, entende-se que o paralelogramo em estudo é $\mathrm{ABCD}$; sua diagonal é AC; os paralelogramos à volta da diagonal são AHKE e KFCG; os complementos dos paralelogramos à volta da diagonal são os quadriláteros EBGK e DHKF. Pela proposição 43, esses quadriláteros (EBGK e DHKF) têm áreas equivalentes.

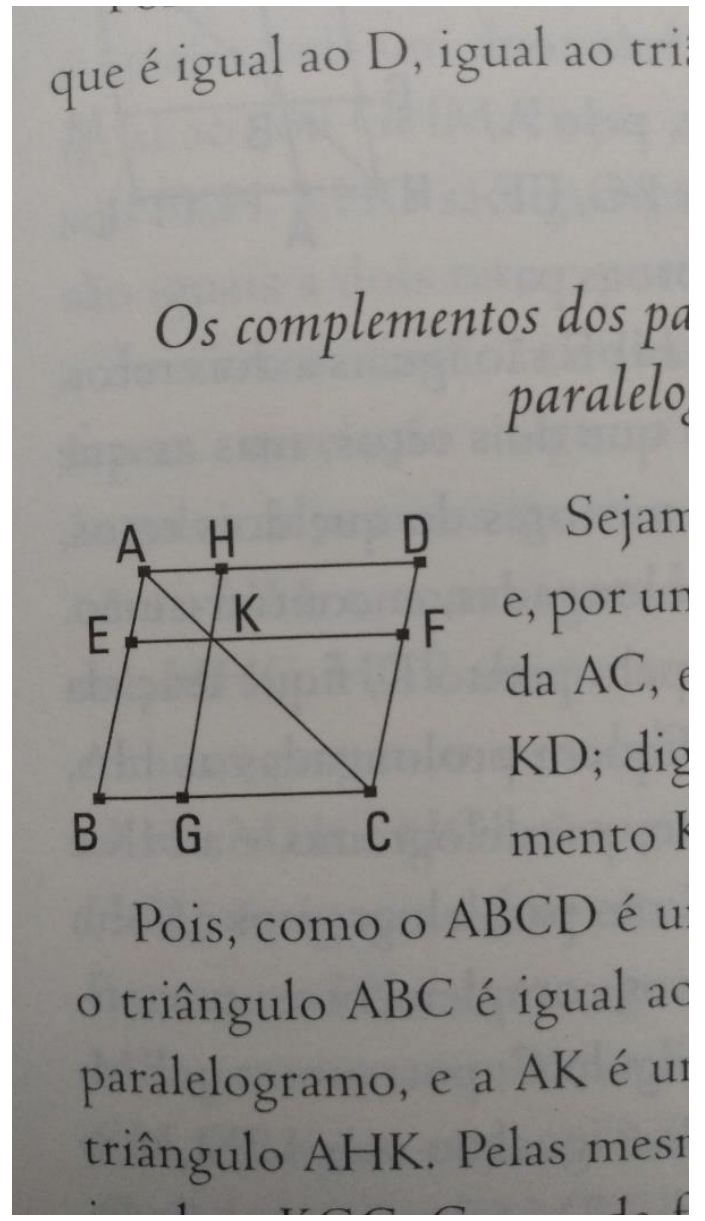

Figura 8: paralelogramo de Os elementos

Fonte: Euclides, 2009, p. 129. 
Agora comparemos a figura de Os elementos (figura 8) com o desenho de Da Vinci (figura 5) ou com os desenhos que obtivemos a partir do procedimento de Da Vinci. É possível verificar que o quadrilátero $\mathrm{ABCD}$ de Os Elementos é semelhante ao construido por Da Vinci (BNOG). No paralelogramo de Da Vinci: a diagonal é NG; os os paralelogramos à volta da diagonal são ANMC e DCEG; os complementos dos paralelogramos à volta da diagonal são os quadriláteros $\mathrm{ABCD}$ e MOEC. Pela proposição 43, esses últimos quadriláteros têm áreas equivalentes, ou seja, o quadrado obtido MOEC tem área equivalente ao retangulo $\mathrm{ABCD}$. Assim, novamente se comprovou o que Da Vinci pretendia provar.

Da Vinci segue seu estudo e cita ainda a definição 2 do livro II de Os elementos, segundo a qual: E, toda área paralelogrâmica, um dos paralelogramos, qualquer que seja, à volta da diagonal dela, com os dois complementos, seja chamado um gnômon (EUCLIDES, 2009, p. 124, 129, 135). Gnômon é a figura obtida pela junção de um dos paralelogramos à volta da diagonal do paralelogramo maior com seus complementos em relação a esse paralelogramo maior. Observando-se a figura 8 , se for retirado o paralelogramo KFCG, obtêm-se o gnômon mencionado. O mesmo pode ser feito no desenho (figura 5) de Da Vinci se for retirado dele o quadrilátero ANMC.

\section{Conclusões}

O estudo da folha 544rv feito neste trabalho nos permite concluir que o objetivo de discutir o método de Leonardo da Vinci para a quadratura do retângulo no Códice Atlântico foi alcançado, pois fizemos a apresentação da folha, a sua descrição, uma tradução do texto contido na folha, explicamos cada passo de Da Vinci na demonstração que ele fez e fizemos observações sobre o método praticado por ele, inclusive, apontando algumas incoerências e suas possíveis causas.

Nosso entendimento é de que este trabalho pode contribuir com o campo da Educação Matemática, em particular o da História da Matemática, pois apresenta um tema de matemática discutido desde a Antiguidade Grega, mas que está contido em um manuscrito do Século XVI pouco explorado em pesquisas Brasileiras, pois na pesquisa mais ampla já mencionada constatamos que há somente um trabalho no Brasil sobre o Códice Atlântico, mas que não discute matemática.

Esperamos que este trabalho possa contribuir para a ampliação dos conhecimentos dos leitores à medida que utilizem os resultados aqui apresentados ou que reflitam sobre suas práticas e/ou pesquisas a partir do que foi discutido neste texto. Como já foi mencionado, este trabalho faz parte de uma investigação mais ampla e apresenta resultados que serão complementados com outras publicações que aprofundarão o tema devido sua amplitude e relevância para o campo de pesquisa. 


\section{Bibliografia}

A VIDA de Leonardo da Vinci. Direção e produção de Renato Castellani. Itália: RAI CBS, 1971. 2 DVD's (270 min).

BAGNI, G. T.; D’AMORE, B. Leonardo e a matemática - São Paulo, SP. Editora Livraria da Física, 2012.

BARROS, J. A. (2004). O campo da história: especialidades e abordagens. Petrópolis: Vozes.

DA VINCI, L. O Códice Atlântico de Leonardo da Vinci. Transcrição e aparatos críticos Augusto Marinoni. Barcelona: Fólio, 2008.

EUCLIDES. 2009. Os elementos. Tradução e introdução de Irineu Bicudo, São Paulo, Editora da UNESP.

ISAACSON, W. Leonardo da Vinci. Tradução de André Czarnobai. 1. Ed. - Rio de Janeiro: Intrínseca, 2017.

KATZ, V. J. História da Matemática. Tradução de Ana Sampaio e Filipe Duarte. Lisboa: Fundação Calouste Gulbenkian, 2010.

KLINE, M. El pensamiento matemático de la Antiguedad a nuestros días, I. Madrid: Alianza Editorial, 1992.

MARTINS, J. P. Uma linguagem geométrica singular refletida no Códice Atlântico de Leonardo da Vinci: contribuições para a geometria escolar. Tese (Doutorado). Universidade Federal do Pará, Instituto de Educação Matemática e Científica, Programa de Pós-Graduação em Educação em Ciências e Matemáticas, Belém, 2021.

MARTINS, J. P. Duplicación del cuadrado y el volumen de sólidos en el Códice Atlántico de Leonardo da Vinci: un estudio de la hoja 100r. Revista Paradigma, V. XLI, No Extra 1, p. $285 \quad-\quad 316,2020 . \quad$ Disponível em: http://revistas.upel.digital/index.php/paradigma/article/view/8574. Acesso em: 14/11/2020.

MARTINS, J. P. MENDES, I A. Informações históricas do Códice Atlântico de Leonardo da Vinci mobilizadas para o ensino de geometria na Educação Básica. Boletim Cearense de Educação e História da Matemática (BOCHEM, V. 07, N. 20, p. 392 - 405, 2020. Disponível em: https://revistas.uece.br/index.php/BOCEHM/article/view/3458 . Acesso em: 28/10/2020.

NAVONI, M. Leonardo da Vinci y los secretos del Códice Atlántico. Tradução Eva María Cantenys Félez e Antonio Díaz Pérez. Barcelona: Art Blume, S. L., 2012.

OZANAM, J. Recreations in mathematics and natural philosophy. recomposed by $\mathrm{m}$. Montucla and tr. by C. Hutton. Publishers: Jean Étienne Montucla, Edward Riddle. 1814 (Do original da Oxford University, digitalizado em 2006). Disponível em: RBHM, Vol. 21, n 41 , pp. 73-88, 2021 
Jeová Pereira Martins \& Iran Abreu Mendes

https://books.google.com.br/books/about/Recreations_in_mathematics_and_natural_p.html ?id=BPIDAAAAQAAJ\&redir_esc=y. Acesso em: 14/06/2020.

SÁNCHEZ, J. L.; ALMARZA, M. O Códice Atlântico de Leonardo da Vinci. Barcelona: Fólio, 2008. (Coleção O códice Atlântico de Leonardo da Vinci, Vol. 10).

WHITE, M. Leonardo o primeiro cientista. 4 ed. - Rio de Janeiro, RJ. Record, 2002.

\section{Jeová Pereira Martins}

Secretaria de Estado de Educação do Pará - SEDUC-PA.

Brasil

e-mail: jeovapereira80@outlook.com

Iran Abreu Mendes
Universidade Federal do Pará - UFPA - Brasil.
Instituto de Educação Matemática e Científica (IEMCI).
e-mail: iamendes1@gmail.com

